\title{
Image analysis of packed beds of spherical particles of different sizes
}

\author{
Manuel Mota, José A. Teixeira, Alexander Yelshin \\ Department of Biological Engineering, University of Minho, 4907 Braga, Codex, Portugal
}

Received 31 July 1997; received in revised form 16 March 1998; accepted 8 May 1998

\begin{abstract}
Two-dimensional simulations of packed beds composed of binary and ternary particle mixtures were made and image analysis of the bed structure was used to determine the bed porosity and tortuosity. Both the porosity and tortuosity were found to be dependent upon the volume fraction of the large particles. However, the volume fraction alone does not totally determine either the porosity or the tortuosity. For a bed of two different sizes of particles, its tortuosity may be considered a product of two quantities, the macro- and micro-tortuosity, each of which can be determined from the corresponding monosized particle beds. (C) 1999 Published by Elsevier Science B.V. All rights reserved.
\end{abstract}

Keywords: Packed bed; Spherical particles; Modelling; Porosity; Tortuosity; Diffusivity

\section{Introduction}

There are several ways of describing porous media and the associated mass transfer phenomena [1-3] occurring in the media. In general, the two major properties are the permeability coefficient (flow phenomena) and the effective diffusion coefficient (mass transfer phenomena). Both coefficients, in turn, are functions of the characteristics of porous media, namely the media porosity $\epsilon$ and tortuosity $T$ [1-3].

The Kozeny-Carman equation which describes the flow of fluids through porous media may be expressed as [1]

$$
u=k \frac{\Delta p}{\mu L}
$$

where $u$ is the fluid velocity, $k$ the medium perme- ability, $\Delta p$ the pressure drop across the medium; $\mu$ the fluid viscosity, and $L$ the medium thickness. The Kozeny coefficient $K$ may be expressed as

$$
K=K_{0} T^{2}
$$

where $K_{0}$ is a constant (equal to 2 in most cases). The permeability $K$ is given as

$$
k=\epsilon^{3} d_{\mathrm{s}}^{2} / 36 K(1-\epsilon)^{2}=\left(\frac{\epsilon}{T}\right)^{2} \frac{\epsilon d_{\mathrm{s}}^{2}}{36(1-\epsilon)^{2} K_{U}}
$$

where $\epsilon$ is the porosity and $d_{s}$ is the equivalent particle diameter.

The effective diffusion coefficient $D_{\mathrm{e}}$, which characterizes mass transfer in porous media, is written as

$$
D_{\mathrm{e}}=D_{0} \frac{\epsilon}{T}
$$


or

$\eta=\frac{D_{\mathrm{e}}}{D_{0}}=\frac{\epsilon}{T}$

where $D_{0}$ is the diffusion coefficient in the bulk medium.

As can be seen, $D_{\mathrm{e}}$ and $K$ depend respectively on and $\epsilon / T(\epsilon / T)^{2}$. Although extensive efforts have been directed toward the study of porous media $[4,5]$, the relationship between the porosity, the tortuosity and the size distribution of particles constituting the media is still open to question. Prediction and control of permeability or effective diffusivity of beds packed with mixtures of particles are important to processes such as catalysis with coke deposition, immobilized cells inside a porous matrix, chromatography, biofilms, deep bed filtration, etc.

In this study, two-dimensional simulation of packed beds formed with binary and ternary particle mixtures were made and image analysis was applied to determine and characterize the media structure. Specifically, the lowest bound for tortuosity and the effect of the volume fraction of the largest particles on the tortuosity, porosity and the ratio $\epsilon / T$ were examined.

\section{Previous studies}

The porosity $\epsilon$ of mixed particle beds has been determined both experimentally and analytically [6-15]. For many of these studies the results obtained were found to be dependent on the manner with which the beds were formed.

Abe and Hirosue [16] presented equations that predict the porosity dependence on the volume fraction of the large particles of the binary mixtures. According to Abe and Hirosue [16], for a binary mixture the dependence of porosity $\epsilon$ on the volume fraction of the large particles $x_{D}$ is represented by two functions (Eq. (6) and Eq. (8)). These two functions form a curve with an intersection point which divides the $x_{\mathrm{D}}-\epsilon$ curve into two parts: part I, the left branch of the curve is given by

$$
\begin{aligned}
& \epsilon=1-\frac{1-\epsilon_{d}}{\left(1-x_{\mathrm{D}}\right)+\alpha x_{\mathrm{D}}\left(1-\epsilon_{d}\right)} \\
& \alpha=1+f_{\mathrm{c}} \cdot(d / D)
\end{aligned}
$$

where $D$ is the large particle diameter of the binary mixture, $d$ is the small particle diameter, $f_{\mathrm{c}}$ is a coefficient and is equal to 1 for $d / D=0.5,1.2$ for $d / D=0.25$ and 1.4 for $d / D \leq 0.125 ; \epsilon_{\mathrm{d}}$ is the porosity of monosized bed of small particles.

The right branch of the $x_{\mathrm{D}}-\epsilon$ curve is given by

$\epsilon=1-\frac{1-\epsilon_{\mathrm{D}}}{x_{\mathrm{D}}\left(1+\beta_{\mathrm{s}} \beta_{\mathrm{c}}\right)^{3}}$

where $\left.\beta_{\mathrm{s}}=(d / D)^{n}, \beta_{\mathrm{c}}=\sqrt{1+\left(1-x_{\mathrm{D}}\right.} / x_{\mathrm{D}}\right) 3-1, n=$ $1 / 2$ for spherical particles and $\epsilon_{\mathrm{D}}$ is the porosity of a monosized bed of large particles.

As the porosity for monosized spherical particles is 0.4 , the minimal porosity for $d / D \rightarrow 0$ will be 0.16 when the volume fraction of large particles in the mixture is $x_{\mathrm{D}}=0.714$. This is the lower bound of the porosity of beds of binary particle mixtures [16]. Ouchiyama and Tanaka [6] compared some published data and their numerical estimations. They found that, for binary mixtures, in the case of $d / D \rightarrow 0$ it is possible to obtain dense packing with a minimal porosity around 0.14 in the region $x_{\mathrm{D}} \sim 0.72$.

The siluation for tortuosity is less clear, because it is difficult to determine $T$ experimentally. Normally, $T$ is calculated through measured values of the porosity and the experimentally determined effective diffusion coefficient or from the Kozeny coefficient.

Several studies gave rise to a wide range of results.

Monte Carlo simulation methods of Knudsen diffusion in a spherical particle bed gave a tortuosity variation of $2.5-5.0$ for different models [17].

Macé and Wei [12] used a model of random walks in a continuum of random spheres and mentioned that tortuosity increases with increasing volume fraction of spheres.

Knudsen diffusion of non-adsorbing gas in a binary mixture of spheres was also considered [18]. Experimental data were interpreted in terms of the 
dependence of the tortuosity on mean pore radius. In all diffusion measurements, a value of 1.48 was found for the tortuosity. Catalyst pellets were simulated by packed beds of microporous ion exchange resin (bead size $0.47 \mathrm{~mm}$ ) and inert glass particles of different size and shape [19]. Both tortuosity and porosity varied with the particle volume fraction and the particle size ratio in the mixture.

The tortuosity of ordered sphere packing was measured experimentally by Olague et al. [20].

The complexity of tortuosity leads to different interpretations of this concept $[1-3,21,22]$. In granular porous media, and their models, the value of tortuosity lies in the region 1.1-1.7. For sintered porous media from metal powder with a particle size range of $40-65 \mu \mathrm{m}$ and $100-200 \mu \mathrm{m}$, the values determined were: for $\epsilon<0.35, T=1.8-2.4 ; \epsilon=$ $0.35-0.4, T=3.63-3.76[23,24]$. Sometimes the definition of tortuosity may be applied to the path of some liquid or objects in a heterogeneous medium. This is the case of bubbles rising in a liquid-solid fluidized bed [25]. The tortuosity of the rise path was defined in this study as the ratio of the total distance to the net vertical distance travelled by a bubble.

Several groups of models concerning tortuosity dependence on porosity have been considered in the literature $[3,22,26,27]$.

From the above mentioned data, it may be seen that tortuosity is a rather complex function of several factors. Some general conclusions can be put forward.

- Tortuosity is evident or latent in all models of mass transfer in porous media.

- Tortuosity is not a physical constant and depends first of all on other porous media characteristics, like porosity, pore diameter, channel shape, etc.

- Tortuosity often depends on processes occurring during mass transfer: porous media compressing or expanding; particles or macromolecules deposition inside pore channel up to pore blocking phenomena; etc.

- Tortuosity depends also on the kind of material being transferred (percolation phenomena). For instance, tortuosity determined for gas diffusion and for macromolecule diffusion through the same porous medium may be different.

\section{Porous media modelling}

Although simplified interpretations of tortuosity have been given in the past, for example as the ratio of channel length to porous medium thickness, estimations of $T$ require the consideration of the effect of factors including the pore length and the pore configuration. This will be briefly discussed below.

\subsection{Effects of pore length}

(1) The simplest case is to consider a pore medium composed of a bundle of capillaries. $T$ is the ratio of the pore length to porous media thickness (Fig. 1(a)). The main feature of this representation is the equidistance of flow streamlines.

(2) Tortuosity of curved pores - this case differs from the previous one by the curvature of the pore channels (Fig. 1(b)). In this case, $T$ increases with the increase in the pore length. The increase in the pore length implies a corresponding increase in the diffusion path of diffusing molecules.

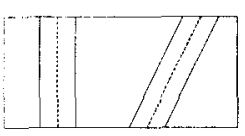

a

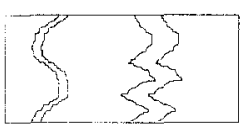

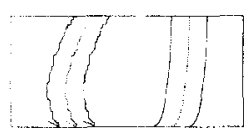

b

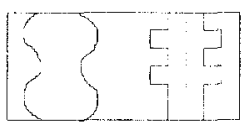

d

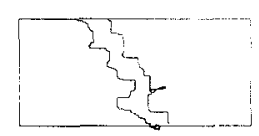

e

Fig. 1. Patterns of pore geometry. 


\subsection{Tortuosity of bent pores}

Usually, for these types of pore, the condition of constant pore diameter or constant cross-section is not valid. The limiting case is a zigzag pore (Fig. 1(c)). The flow streamlines are not equidistant. With bent pores, the inertial and the constriction effects may become significant for macromolecular diffusion. It must be stressed that the pore hydraulic resistance and the effective diffusion do not depend on the pore length only. Indeed, as may be seen from Fig. 2, exactly the same path length may lead to quite different values of tortuosity. The conventional definition $T=L_{\mathrm{e}} / L$ is not always valid. The dependence of $T$ on additional parameters, such as the linear density of bends or the fractal dimension, must be considered. One may even speculate that the constriction effect observed in macromolecular diffusion for small values (molecule size)/(pore size) may be caused by the pore curvature.

\subsection{Pore configuration}

Several kinds of configurations may be found for pores.

(1) Symmetrical straight pores with variable
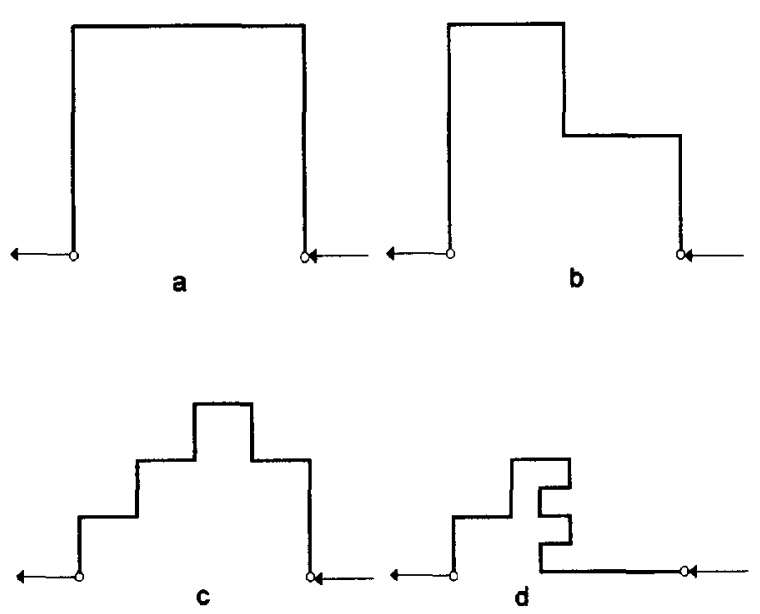

Fig. 2. Different types of pore with uniform thickness, having the same inlet and outlet points (marked by arrows) and the same pore length but a different number of bends arising from different tortuosity: (a) two bends; (b) four bends; (c) eight bends and (d) 11 bends. cross-sectional area. Although the pore may have a length equal to the porous medium thickness, the average path of a molecule or stream is not equal to the medium thickness. In this case, pores can be characterized by a ratio of largest to smallest diameters or cross-sectional area, or by means of stream trajectories. For this type of pore, several effects can be expected: inertia; constriction related to curvature of trajectories; constriction related to change of pore cross-section area.

(2) Symmetrical bent pores with variable crosssectional area. Pore bending increases the first two effects described previously.

(3) Goffered pores. In this case, a new effect characterized by the amplitude and frequency of goffers is added to the above-mentioned effects. A regular goffered pore type is obtained if the frequency of pore wall bends increases.

(4) Random pore channels (Fig. 1(e))-the most complicated type of pore, with random configuration of channel walls and random variation of channel length.

The previous analysis highlights the complexity of real porous media. For a better understanding of the tortuosity impact on mass transfer and separation processes it is necessary to investigate simplified porous media models. Binary and ternary mixed beds of spherical particles will thus be the mixtures under consideration. Porous media parameters will be used for their adequacy to this work.

In this work, the ranges of particle diameter ratio were chosen as $D / d=2-15$ and $D / d=2-10$ for binary and ternary mixtures respectively.

\subsection{Modelling of packed beds}

To estimate the porosity, the tortuosity and the ratio $\epsilon / T$ of porous media of binary and ternary particle mixtures with different volume fractions of the largest particles, two-dimensional simulations of medium formation were made. The simulation procedure was similar to those of Suzuki et al. [8] and Tory et al. [28] with minor modifications.

Sets of $N$ particles (corresponding to discs in two dimensions) of a given size were used. For instance, for $n$ large and $m$ small discs then $N=$ $n+m$ ( $n$ and $m$ are integers). The chamber is 
divided in several dropping channels. The channel through which each disc is dropped is randomly chosen. The width of the chamber was 750 pixels. Each falling disc either comes to rest at the bottom of the chamber or will come in contact with one of the particles which is already packed in the bed. After contact, the disc is, if necessary, manually moved to the final position in order to obtain a compact packing. This corresponds to a stabilization of real packing by shaking. In the simulations used, the procedure stopped when a total number of 1000 particles were attained. Calculations of both volume and void fractions of largc particles in the mixture were done by image analysis.

The tortuosity estimation was also done by image analysis. The trajectory of a falling test point (pixel) was established on the basis of the following assumptions: the test point can only move downwards; in branching points the test point moves to the branch chosen on the basis of a minimal trajectory length. This corresponds to a lower tortuosity bound, which was calculated as the ratio of the estimated trajectory length of the test point to the height of the layer, that is

$$
\begin{aligned}
& T=\sum_{i=1}^{N} \sqrt{\left(x_{i}-x_{i-1}\right)^{2}+\left(y_{i}-y_{i-1}\right)^{2}} / \sum_{i=1}^{N} \\
& \times\left(y_{i}-y_{i-1}\right)
\end{aligned}
$$

where $x_{i}$ and $y_{i}$ are projections of straight bends on axis $x$ and $y$ respectively, and $N$ is the total number of segments in the bent line.

For each packing, the mean value of the tortuosity was determined with six test points. With this approach, the average two-dimensional tortuosity $T_{0}$ for a monosized spherical particle bed is $T_{0}=1.1547$.

The ternary mixture model is built by filling the void space of the binary mixture with smaller discs. Calculations were made as previously described for binary mixtures. Typical examples of twodimensional charts of binary and ternary mixtures are shown in Fig. 3.

Results for three types of binary bed of particle size ratios in the mixture $n=D / d=21 / 11=1.91$, $D / d=31 / 8=3.875$ and $D / d=63 / 4=15.75$ are presented on Figs. 49.

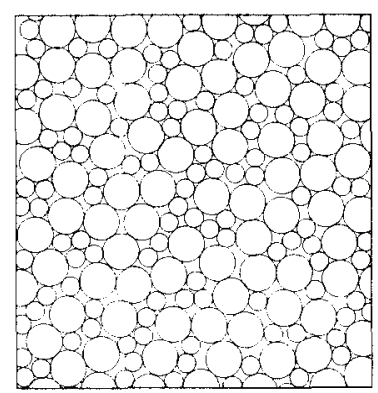

(a)

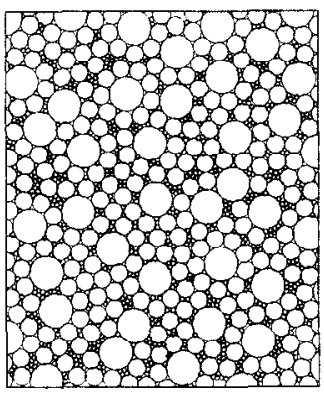

(b)
Fig. 3. Typical porous media models for binary (a) and ternary (b) mixtures. (a) $n=21 / 11=1.91, \quad x_{\mathbf{D}}=0.727, \epsilon / \epsilon_{0}=$ $0.776, T / T_{0}=1.088$ and $\eta / \eta_{0}=0.713$. (b) $m=1.91, n=7.78$, $x_{\mathrm{D}}=x_{1}=0.3, x_{2}=0.11, x_{3}=0.5935, \epsilon / \epsilon_{0}=0.314, \quad T / T_{0}=1.04$ and $\eta / \eta_{0}=0.305$.

\section{Discussion}

The discussion below aims at establishing boundary estimations for normalized porous media characteristics: $\epsilon, T$ and $\eta=\epsilon / T$.

\subsection{Porosity}

The variation of normalized porosity for binary mixture on $x_{\mathrm{D}}$, Fig. 4 , is compared with the dependence described by Abe and Hirosue [16].

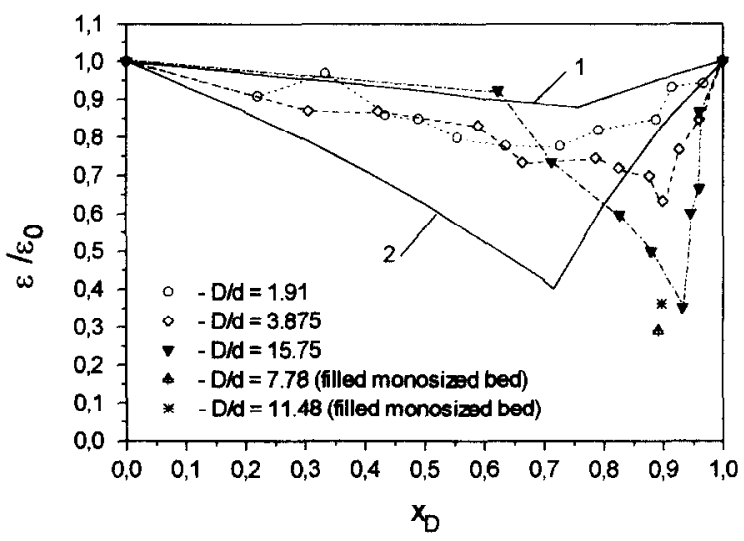

Fig. 4. Dependence of normalized porosity $\epsilon / \epsilon_{0}$ on $x_{\mathrm{D}}$ for different particles size ratio $n=D / d$. 1 and 2 are predictions by Eqs. (6) and (8). Curve $1, \epsilon_{0}=\epsilon_{\mathrm{d}}=\epsilon_{\mathrm{D}}=0.4$ and $D / d=2$, and curve 2 , the same for $(D / d)^{-1} \rightarrow 0$. Points are simulation results for a binary mixture; $\epsilon_{0}$ is the porosity of a bed of monosized particles, $\epsilon_{0}=0.407$. 
However, the minimal porosity value was found at a higher value of $x_{\mathrm{D}}$ than the prediction by Abe and Hirosue [16]. It is important to notice that for large $D / d$ ratios a minimal porosity is obtained for $x_{\mathrm{D}}$ values in the range 0.85 to 0.95 . On the other hand, these results indicate the possibility of creating denser packings than that predicted theoretically (Eqs. (6) and (8)).

A ternary particle bed was created by filling the void space of the binary particle bed, mentioned above, with small particles of diameter 2.7. The results of $\epsilon / \epsilon_{0}$ versus $x_{\mathrm{D}}$ are shown in Fig. 5. The points marked with arrows belong to binary particle beds formed by filling monosized particle beds of: $D=11$ and $D=8$ (left arrows), and $D=21$ and $D=31$ (right arrows).

The maximum fine particle volume fraction placed in the void space of binary particle beds ranged from 6 to $11 \%$. From Fig. 5 it can be seen that the region of minimal porosity for dense packing is broader than in the case of binary mixtures (Fig. 4).

According to $\mathrm{Yu}$ and Standish [11] the maximal packing density for ternary particle mixtures of particle size ratio $31: 8: 2.7$ should be $\epsilon / \epsilon_{0}=0.5$. As a matter of fact, this simulation shows that a much lower value for $\epsilon / \epsilon_{0}$ (e.g. 0.1 , see Fig. 5) may be obtained.

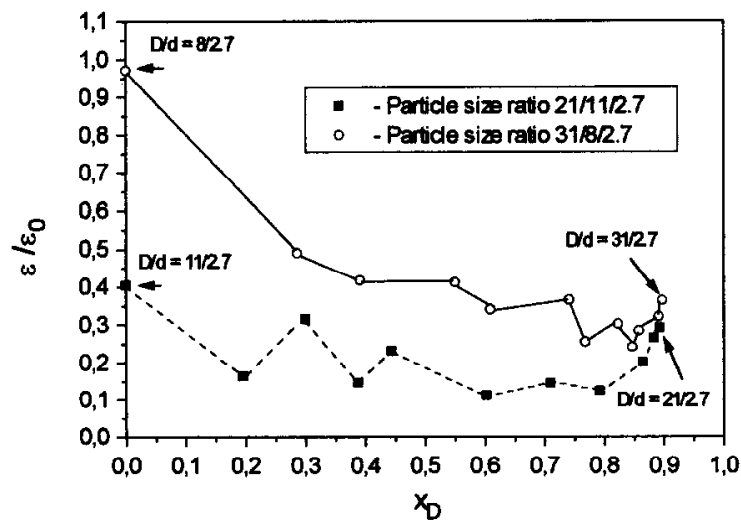

Fig. 5. Variation of $\epsilon / \epsilon_{0}$ with $x_{\mathrm{D}}$ in ternary mixtures. Mixtures were created by filling the void space of binary mixtures composed either of particles of a diameter ratio $n=D / d=21 / 11=$ 1.91 or $n=D / d=31 / 8=3.875$ with small particles with diameter 2.7.

\subsection{Tortuosity}

The maximum tortuosity (Fig. 6) does not correspond to the minimal porosity (Fig. 4). This means that the tortuosity must not only be a function of the porosity but also of porous medium topology.

For ternary particle beds the tortuosity increases monotonically with the increase in $x_{\mathrm{D}}$ and reaches a maximum when the volume fraction of intermediate particles approaches zero (Fig. 7). Points marked with arrows belong to binary mixtures created by filling monosized beds of particles $D=$

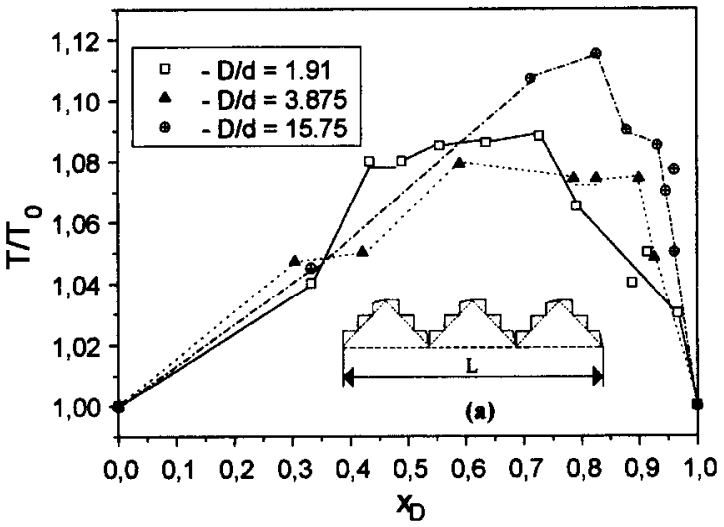

Fig. 6. Dependence of binary mixture normalized tortuosity $T / T_{0}$, on volume fraction $x_{\mathrm{D}}$ for different ratios $n=D / d$; (a) represents a test point pathway for maximal tortuosity; $T_{0}$ is the tortuosity of monosized particle layer, $T_{0}=1.1547$.

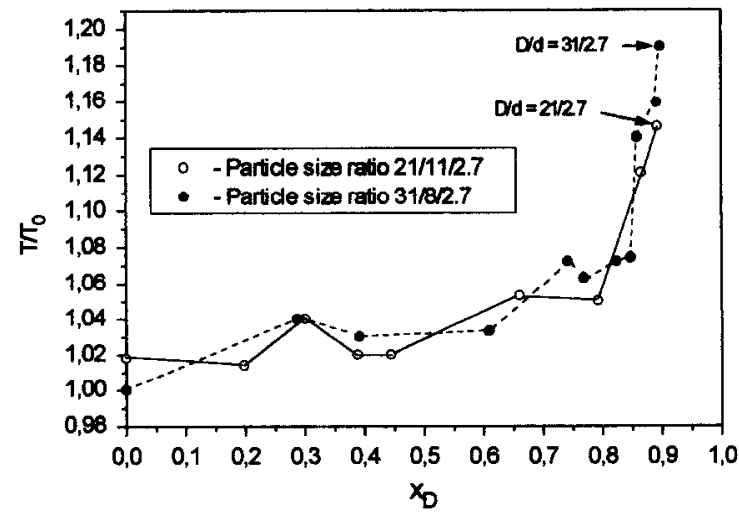

Fig. 7. Variation of $T / T_{0}$ with $x_{\mathrm{D}}$ for diameter ratios $21: 11: 2.7$ and 31:8:2.7 in ternary mixtures. $T_{0}$ is the porosity of monosized particles layer, $T_{0}=1.1547$. 
21 and $D=31$ when the volume fraction of the intermediate particles equals zero.

It is possible to consider tortuosity of mixed binary beds as a combination of two components.

The first component of the total tortuosity $T$ represents tortuosity generated in the bed void space by the large particle skeleton $T_{\mathrm{D}}$. This will be called the macro-tortuosity. The second component of $T$ represents a tortuosity generated by the small particles filling the skeleton's void space. This will be called the micro-tortuosity $T_{\mathrm{d}}$. Accordingly, the test point pathway will be as shown in Fig. 6(a). The total pore length is equal to $L_{\mathrm{e}}$ (full broken line on Fig. 6(a)). The length of the line, which corresponds to the macro-tortuous path scale (dotted curve on the scheme), is $L_{\mathrm{D}}$. Hence

$T_{\mathrm{d}}=L_{\mathrm{e}} / L_{\mathrm{D}}$

$T_{\mathrm{D}}=L_{\mathrm{D}} / L$

where $L$ is the bed thickness.

The total tortuosity $T$, according to Eqs. (10) and (11) can be written as

$T=\frac{L_{\mathrm{e}}}{L}=\frac{L_{\mathrm{D}} T_{\mathrm{d}}}{L}=\frac{L T_{\mathrm{D}} T_{\mathrm{d}}}{L}=T_{\mathrm{D}} T_{\mathrm{d}}$

Since in both cases the tortuosity $\left(T_{\mathrm{D}}\right.$ and $\left.T_{\mathrm{d}}\right)$ is generated by spherical particles, we can assume for large values of $D / d$ in the range of maximal tortuosity $T$, that $T_{\mathrm{D}} \approx T_{\mathrm{d}}=T_{0}$, where $T_{0}$ is the tortuosity of monosized spherical particle beds. Hence, in our case of the two-dimensional model, $T_{0}=1.1547$ as previously mentioned and

$T=T_{0}^{2} \quad$ or $\quad \frac{T}{T_{0}}=1.1547$

which is in good agreement with maximal normalized tortuosity $T / T_{0}=1.12$, measured for $D / d=$ 15.75 (Fig. 6).

Since this estimation was made for the minimal pathway in the mixed bed, we can use it as a low tortuosity bound for two-dimensional mixed beds when $D / d \rightarrow \infty$.

This approach can be extended to a ternary mixture if the extra tortuosity generated by inter- mediate particle fraction is assumed to be $T_{\mathrm{i}}$ :

$T=T_{\mathrm{D}} T_{\mathrm{i}} T_{\mathrm{d}}$

Then, for $T_{0}=1.1547$ we have for $D / d \gg 15$

$T=T_{0}^{3} \quad$ or $\quad \frac{T}{T_{0}}=T_{0}^{2}=1.333$

When $D / d \gg 15$, the diameter of small particles tends to zero and the ternary mixture will approach a binary mixture. This means that 1.333 will be the highest possible value for the lower bound of tortuosity of ternary mixtures.

\subsection{Ratio $\eta=\epsilon / T$}

The ratio $\eta=\epsilon / T$, which is used as a diffusivity coefficient $\eta=D_{\mathrm{e}} / D_{0}$, or $\eta^{2}=(\epsilon / T)^{2}$ which is present in the permeability expression (Eq. (3)), depends on $x_{\mathrm{D}}$. This raises the possibility of controlling mass transfer properties of packed beds (see Figs. 8 and 9). The comparison between the observed variation of porosity and tortuosity values shows that, for this particular case of dense packing, porosity is more affected than tortuosity, by either the volume fraction $x_{\mathrm{D}}$ or the particle diameter ratio. As a conscquence, the behaviour of the ratio $\eta=\epsilon / T$ is similar to that observed for the dependence of porosity on $x_{\mathrm{D}}$.

Finally, for binary packing, it must be pointed out that two different packings may have the same porosity, tortuosity or value of $\eta$ and a quite

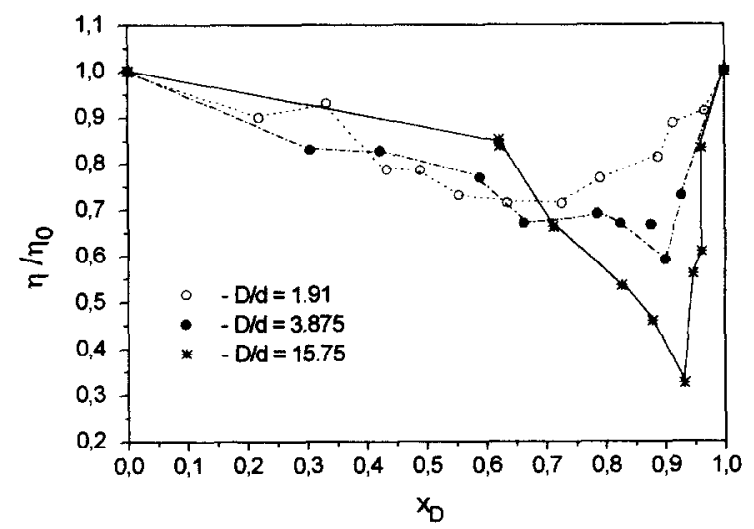

Fig. 8. Variation of $\eta / \eta_{0}$ with $x_{\mathrm{D}}$ for different $n=D / d . \eta=\epsilon / T$ and $\eta_{0}=\epsilon_{0} / T_{0}$, in binary mixtures. 

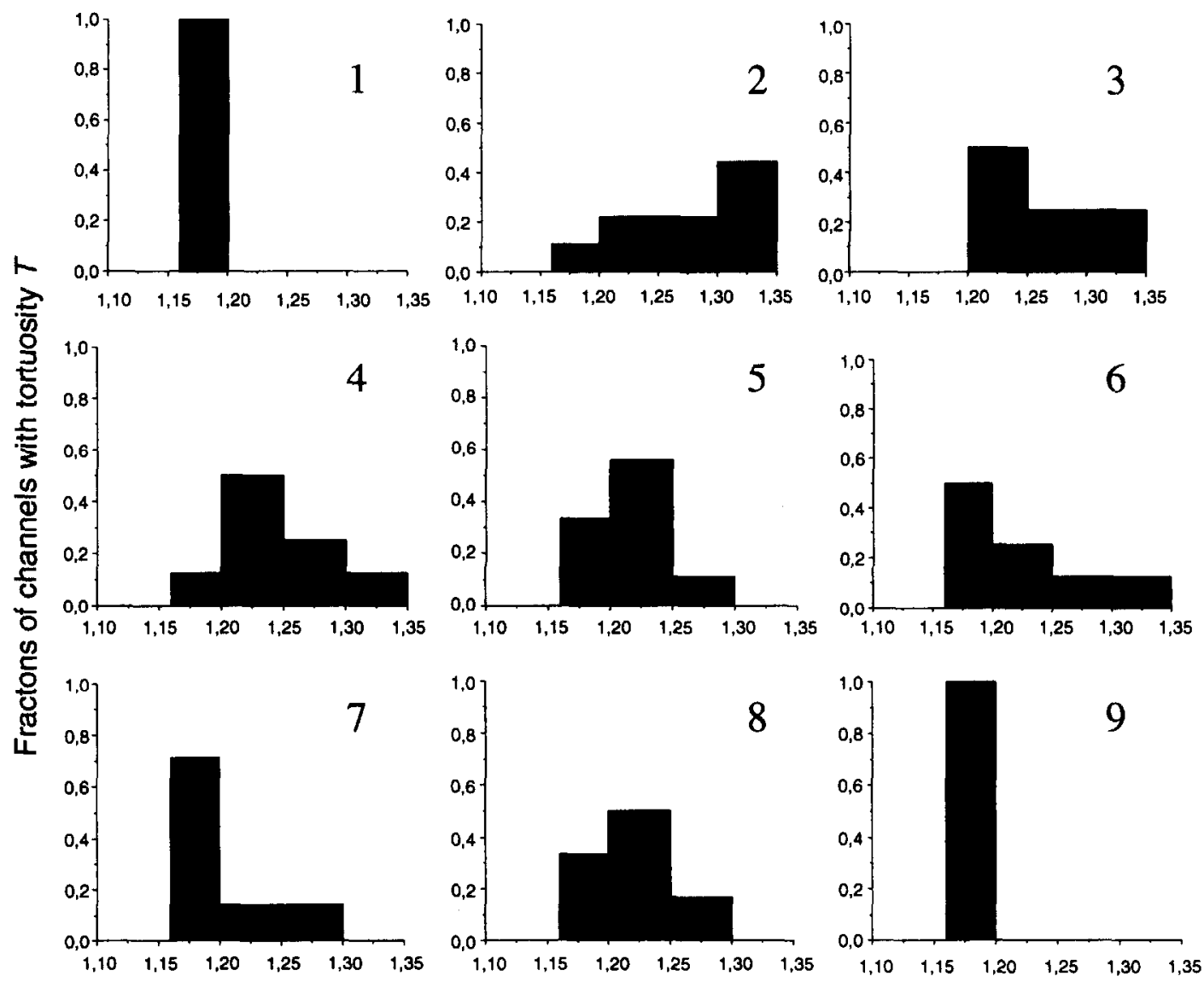

Tortuosity; $T$, range

Fig. 9. Measured tortuosity distribution for $D / d=1.91$. (1) $x_{\mathrm{D}}=0.0$. (2) 0.49 , (3) 0.555 , (4) 0.636 , (5) 0.727 , (6) $0.7916,(7) 0.888$, (8) 0.914 , and (9) 1.0. $x_{\mathrm{D}}$ values between 0.6363 and 0.727 correspond to maximal tortuosity values.

different volume fraction. This fact must be taken into consideration when binary packing data are represented in terms of $T$ versus $\epsilon$.

The main effect on tortuosity in mixed beds comes from the topology of porous media. For instance, there is a significant effect on tortuosity by increasing the number of bends in porous medium channels, especially in the case of ternary mixtures. Fig. 9 displays the tortuosity distribution measured by image analysis for $D / d=1.91$, corresponding to nine different $x_{\mathrm{D}}$ values.

As we can see, in mixtures close to monosized beds $\left(x_{\mathrm{D}} \rightarrow 0\right.$ or $\left.x_{\mathrm{D}} \rightarrow 1\right)$ the structure gives rise to an almost regular packing, pores tend to be of regular shape and the tortuosity range will be narrow owing to the uniform length of pores. Therefore the $T$ variation is small. On the other hand, the distribution of tortuosity is broader for intermediate $x_{D}$ values, indicating a non-uniform distribution of pore lengths, and the average tortuosity increases (Fig. 6).

The same reasoning which was applied for the effect of porosity on $\eta=\epsilon / T$ in binary mixtures can also be used for ternary mixtures. It may be seen that a fraction as small as $6-10 \%$ of fine particles in the mixture can reduce the diffusivity coefficient by two- to four-fold or more (Fig. 10). This means the fraction of the finest particles in packed beds may drastically effect mass transfer phenomena in porous media. 


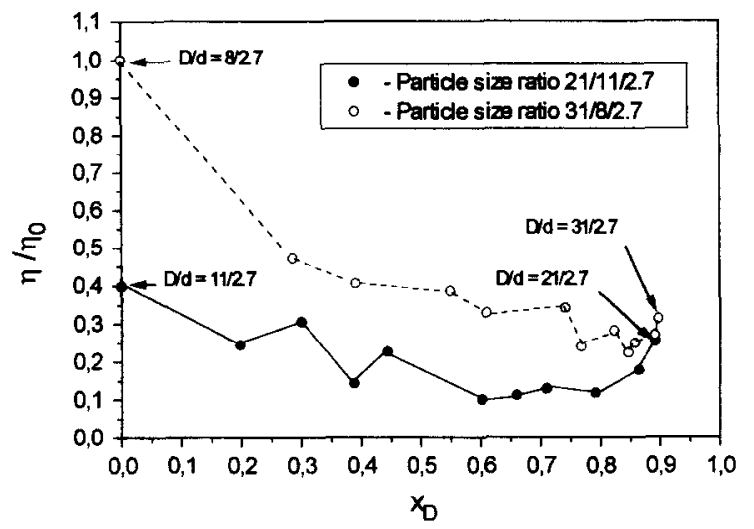

Fig. 10. Variation of $\eta / \eta_{0}\left(\eta=\epsilon / T, \eta_{0}=\epsilon_{0} / T_{0}\right)$ with $x_{\mathrm{D}}$, for ternary mixtures.

\section{Conclusion}

Two-dimensional image modelling of binary and ternary mixed porous media constructed with spherical particles gave the possibility of getting lower bound values for porosity, tortuosity and the ratio $\eta=\epsilon / T$. Different volume fractions of large and small particles in the mixture, as well as different ratios of particle size, were investigated.

The tortuosity dependence on volume fraction of large particles passed through a maximum, which did not correspond to the minimum value of the porosity. The introduction of a third fraction of smaller particles into the binary model led to a dramatic decrease in porosity. For a volume fraction of smaller particles as low as $0.06-0.1$, porosity decreased to $20-30 \%$ of the corresponding porosity value for monosized porous media. This deviation increased by increasing the particles size ratio.

These results call attention to the importance of the presence of a very small fraction of dust particles in the transport characteristics of a porous medium.

It was proposed that tortuosity could be decomposed in micro- and macro-tortuosity. As was demonstrated, total tortuosity is the product of micro- and macro-tortuosity. The calculated results are in good agreement with experimental results for binary mixtures. The concept was generalized to ternary mixtures.

The characteristics of a mixed bed must there- fore take into account other properties besides particle size. Different beds with a similar equivalent particle diameter can have quite different values of both porosity and tortuosity.

The ratio $\epsilon / T=D_{\mathrm{e}} / D_{0}$ also passed through a minimum. Therefore, an $x_{\mathrm{D}}$ increase may decrease the effective diffusion coefficient to $70 \%$ of monosized porous medium for a binary mixture and up to $85 \%$ for a ternary mixture.

\section{Acknowledgment}

The financial assistance of the JNICT of Portugal through the program PRAXIS XXI is acknowledged. This work was funded by PRAXIS XXI under grant number BBC/6440/95. The authors also thank Filomena Louro for the help given to the final revision of the English version of this manuscript.

\section{Appendix}

\subsection{Nomenclature}

$D \quad$ largest particles diameter in the mixture

$D_{0} \quad$ diffusion coefficient in bulk liquid

$D_{\mathrm{e}} \quad$ effective coefficient of diffusion

$d \quad$ small particle diameter in the binary mixture

$d_{\mathrm{m}} \quad$ diameter of intermediate particles in the ternary mixture

$d_{\mathrm{n}} \quad$ diameter of smallest particles in the ternary mixture

$d_{s} \quad$ equivalent particle diameter

$K \quad$ Kozeny coefficient

$K_{0} \quad$ Kozeny constant

$k$ permeability $\left(\mathrm{m}^{2}\right)$

$L \quad$ straight line length connecting the two ends of a tortuous pore or layer thickness

$L_{\mathrm{e}} \quad$ tortuous pore length

$m \quad$ ratio $D / d_{\mathrm{m}}$

$n \quad$ ratio $D / d$ for binary mixture and $n=D / d_{n}$ for ternary mixture

$T \quad$ tortuosity

$T_{0} \quad$ tortuosity of monosized bed

$T_{\mathrm{D}} \quad$ macro-tortuosity

$T_{\mathrm{d}} \quad$ micro-tortuosity

$u \quad$ flow velocity $\left(\mathrm{m} \mathrm{s}^{-1}\right)$

$x_{1} \quad$ volume fraction of largest particles in the ternary mixture $\left(x_{1}=x_{\mathrm{D}}\right)$

$x_{2} \quad$ volume fraction of smallest particles in the ternary mixture 
$x_{3} \quad$ volume fraction of intermediate particles in the ternary mixture

$x_{\mathrm{D}} \quad$ volume fraction of large particles in the mixture

$\Delta p \quad$ pressure drop $(\mathrm{Pa})$

\subsubsection{Greek letters}

$\epsilon \quad$ porosity of mixed bed

$\epsilon_{0} \quad$ porosity of monosized bed

$\epsilon_{\mathrm{D}} \quad$ porosity of large particle monosized bed

$\epsilon_{\mathrm{d}} \quad$ porosity of small particles layer

$\phi \quad$ packing density

$\eta \quad$ ratio $(\eta=\epsilon / T)$

$\eta_{0} \quad$ ratio $\left(\mathrm{I}_{0}=\epsilon_{0} / T_{0}\right)$

$\mu \quad$ fluid viscosity

\section{References}

[1] J. Bear, Dynamics of Fluids in Porous Media, Elsevier, New York, 1972.

[2] F.A.L. Dullien, Single phase flow through porous media and pore structure, Chem. Eng. J. 10 (1975) 1-34.

[3] M. Suzuki, Adsorption Engineering, Elsevier, 1990.

[4] A.B. Yu, R.P. Zou, N. Standish, Modifying the linear packing model for predicting the porosity of nonspherical particle mixtures, Ind. Eng. Chem. Res. 35 (1996) 3730-3741.

[5] R.P. Zou, A.B. Yu, Evaluation of the packing characteristics of mono-sized non-spherical particles, Powder Technol. 88 (1996) 71-79.

[6] N. Ouchiyama, T. Tanaka, Porosity of mass of solid particles having a range of sizes, Ind. Eng. Chem. Fundam. 20 (1981) 66-71.

[7] Z.Z. Hulewicz, Resistance to flow through a granular bed in the laminar regime. I. Derivation of a new correlating equation, Int. Chem. Eng. 27 (1987) 566-573.

[8] M. Suzuki, K. Makino, M. Yamada, K. Ionoyo, A study on the coordination number in a system of randomly packing, uniform-sized spherical particles, Int. Chem. Eng. 21 (1981) 482-488.

[9] K. Gototh, T. Chuba, A. Suzuki, Computer simulation of weight distributions of spherical-particles beds on the bottom of a container, Int. Chem. Eng. 22 (1982) 107-115.

[101 M. Kuramae, Investigation of unsaturated liquid flow in a granular bed using a cubic-lattice pore model, Int. Chem. Eng. 22 (1982) 666-673.

[11] A.B. Yu, N. Standish, Estimation of the porosity of particle mixtures by a linear-mixture packing model, Ind. Eng. Chem. Res. 30 (1991) 1372-1385.

[12] O. Macé, J. Wei, Diffusion in random particle models for hydrodemetalation catalysts, Ind. Eng. Chem. Res. 30 (1991) 909-918.

[13] M.J. MacDonald, C.F. Chu, P.P. Guilloit, K.M. Ng, A generalized Blake-Kozeny equation for multisized particles, AIChE J. 37 (1991) 1583-1588.

[14] C.Y. Lin, J.C. Slattery, Three-dimensional, randomised, network model for two-phase flow through porous media, AIChE J. 28 (1982) 311-324.

[15] N. Ouchiyama, T. Tanaka, Porosity estimation for random packings of spherical particles, Ind. Eng. Chem. Fundam. 23 ( 1984) 490-493.

[16] E. Abe, H. Hirosue, Porosity estimation of mixed cake in body filtration, J. Chem. Eng. Jpn. 15 (1982) 490-492.

[17] M.H. Abbasi, J.W. Evans, I.S. Abramson, Diffusion of gases in porous solids: Monte Carlo simulation in the Knudsen and ordinary diffusion regimes, AIChE J. 29 (1983) 617-624.

[18] T. Wright, D.M. Smith, D.L. Stermer, Knudsen diffusion in bidisperse mixtures of uniform spheres, Ind. Eng. Chem. Res. 26 (1987) 1227-1232.

[19] K. Klusácek, P. Schneider, Effect of size and shape of catalyst microparticles on pellet pore structure and effectiveness, Chem. Eng. Sci. 36 (1981) 523-527.

[20] N.E. Olague, D.M. Smith, M. Ciftcioglu, Knudsen diffusion in ordered sphere packings, AIChE J. 34 (1988) 1907-1909.

[21] C.C. McCune, H.S. Fogler, W.E. Kline, An experimental technique for obtaining permeability-porosity relationships in acidized porous media, Ind. Eng. Chem. Fundam. 18 (1979) 188-191.

[22] T.C. Zhang, P.L. Bishop, Evaluation of tortuosity factor and effective diffusivities in biofilms, Water Res. 28 (1994) 2279-2287.

[23] V.K. Sorokin, Correlation of gas permeability with pore structure of metal leafs, Mach. Build. 9 (1976) 129-132.

[24] V.K. Sorokin, V.A. Khrenov, Filtration and mechanical properties prediction of porous stainless steel, Powder Metall. 9 (1984) 88-89.

[25] K. Tsuchiya, A. Furumoto, Tortuosity of bubble rise path in a liquid-solid fluidized bed: effect of particle shape, AIChE J. 41 (1995) 1368-1374.

[26] M.R. Riley ct al., A simple corrclation for predicting effective diffusivity in immobilized cell systems, Biotech. Bioeng. 49 (1996) 223-227.

[27] P.R. Johnston, Determining the size of the average pore from fluid permeability measurements, J. Test. Eval. 11 (1983) 122-125.

[28] E.M. Tory, B.H. Church, M.K. Tam, M. Ratner, Simulated random packings of equal spheres, Can. J. Chem. Eng. 51 (1973) 484. 